\title{
SUBANALYTIC FUNCTIONS
}

\author{
ADAM PARUSIŃSKI
}

\begin{abstract}
We prove a strong version of rectilinearization theorem for subanalytic functions. Then we use this theorem to study the properties of arc-analytic functions.
\end{abstract}

\section{INTRODUCTION}

The main purpose of this paper is to show a strong version of rectilinearization theorem for subanalytic functions (Theorem 2.7 below). Our original aim of introducing this theorem was the proof of existence of Lipschitz stratification for subanalytic sets [P] but it turned out that it can be also used to study arc-analytic functions. Therefore, we have decided to state it separately.

Our approach to subanalytic geometry is based mostly on [H1], where the two major techniques used are desingularization and the local flattening theorem. To make our considerations elementary one may use instead of Hironaka's desingularization various theorems of Bierstone and Milman [BM1]. Also the local flattening theorem can be replaced by a weaker result (Corollary 1.2 below), where instead of flatness we required only equidimensionality of the morphism. We present a short proof of this result in $\S 1$. In $\S 2$ we state and give a proof of our rectylinearization theorem which we use in $\S 3$ to study the properties of arc-analytic functions.

This paper is a corrected version of MPI/90-58 preprint with the same title.

\section{LOCAL BLOWINGS-UP}

Let $f: X \rightarrow M$ be a morphism of complex analytic spaces. We assume for simplicity that $M$ is nonsingular. Let $s_{\alpha}: W_{\alpha} \rightarrow M$ be a composition of local blowings-up with smooth and nowhere dense centers (we refer the reader to [HLT, §3] or [H1, §2] for major properties of this notion). Let $E_{\alpha} \subset W_{\alpha}$ denote the union of the corresponding exceptional divisors. By the strict transform of $f$ by $s_{\alpha}$ we mean the pair $\left(X_{\alpha}, f_{\alpha}\right)$, where $X_{\alpha}$ is the smallest analytic subspace of the fibre product $X \times_{M} W_{\alpha}$ which contains $X \times_{M}\left(W_{\alpha} \backslash E_{\alpha}\right)$ and $f_{\alpha}: X_{\alpha} \rightarrow W_{\alpha}$ is induced by the projection. Let us denote the other projection $X_{\alpha} \rightarrow X$ by $\tilde{s}_{\alpha}$. The following result follows easily from the local flattening theorem ([HLT, Théorème 4] or [H1, Theorem 4.4]).

Received by the editors February 24, 1991 and, in revised form, April 23, 1992.

1991 Mathematics Subject Classification. Primary 32B20.

Key words and phrases. Subanalytic function, rectilinearization, local flattening theorem, arcanalytic functions. 
Theorem 1.1. Let $f: X \rightarrow M$ be a morphism of complex analytic spaces and assume that $M$ is nonsingular. Let $L$ and $K$ be compact subsets of $X$ and $M$ respectively. Then, there exists a finite number of analytic morphisms $s_{\alpha}: W_{\alpha} \rightarrow$ $M$ such that:

(1) each $s_{\alpha}$ is the composition of a finite sequence of local blowings-up with smooth nowhere dense centers;

(2) for each $\alpha$ there is a compact subset $K_{\alpha}$ of $W_{\alpha}$ and

$$
\bigcup_{\alpha} s_{\alpha}\left(K_{\alpha}\right)=K
$$

(3) the strict transforms $f_{\alpha}: X_{\alpha} \rightarrow W_{\alpha}$ are flat at every point $x \in X_{\alpha}$ corresponding to $L$ (i.e., at every $\left.x \in \tilde{s}_{\alpha}^{-1}(L)\right)$.

Remarks. (a) To have the centers nonsingular we need some kind of desingularization theorem. Since the statement already involves local blowings-up it is enough to use Theorem 4.4 of [BM1] (see the end of the proof of Corollary 1.2).

(b) In the original statement of the local flattening theorem $L \subset f^{-1}(y)$ for some $y \in M$. The case of arbitrary compact $L$ can be obtained by a simple topological argument (similar to that of the Topological Remark below).

The local flattening theorem was used mainly to study the properties of subanalytic sets (see, for instance, [H1, H3 or BM2]). In fact for this purpose it is enough to have a weaker result with equidimensionality instead of flatness. We present a short proof of such a result. The idea of proof is similar to that in [B] (one can also use the approach of [DD]).

Corollary 1.2. Let $f: X \rightarrow M, L, K$ be as in Theorem 1.1. Then, there exists a finite number of maps $s_{\alpha}: W_{\alpha} \rightarrow M$, satisfying (1) and (2) of Theorem 1.1 and

$\left(3^{\prime}\right)$ the strict transforms $f_{\alpha}: X_{\alpha} \rightarrow W_{\alpha}$ satisfy at every point $x \in X_{\alpha}$ corresponding to $L$ the following strong equidimensionality property:

$$
\operatorname{dim}\left(f_{\alpha}^{-1}\left(f_{\alpha}(x)\right) \cap X_{\alpha}^{\prime}, x\right)=\operatorname{dim}\left(X_{\alpha}^{\prime}, x\right)-\operatorname{dim} M .
$$

for every irreducible component $X_{\alpha}^{\prime}$ of $X_{\alpha}$ at $x$.

It is enough to check the strong equidimensionality (defined above) on every "pure dimensional" component of $X_{\alpha}$ at $x$. We note also that, like flatness, strong equidimensionality is an open property (in $X_{\alpha}$ ) and implies the universal openness (compare [H3, §4]).

\section{Proof of Corollary 1.2.}

Topological Remark. We start with a simple topological observation which will allow us to proceed by induction. Assume that we have constructed a family $t_{\beta}: V_{\beta} \rightarrow M, K_{\beta} \subset V_{\beta}$, satisfying the conditions (1) and (2) of the statement of Theorem 1.1. Let $f_{\beta}: X_{\beta} \rightarrow V_{\beta}$ denote the corresponding strict transforms and $\tilde{t}_{\beta}: X_{\beta} \rightarrow X$ the induced maps. To proceed by induction we would like to have $\tilde{t}_{\beta}^{-1}(L)$ compact, which is not always the case. Nevertheless, the projection of $\tilde{t}_{\beta}^{-1}(L)$ on $V_{\beta}$ is proper. Therefore, we can fix, for each $\beta$, a relatively compact subset $U_{\beta}$ of $V_{\beta}$ which contains $K_{\beta}$. Then the set $L_{\beta} \subset X_{\beta}$ of points of $f_{\beta}^{-1}\left(\overline{U_{\beta}}\right)$ corresponding to $L$ is compact. 
Assume that for each $\left(f_{\beta}, L_{\beta}, K_{\beta}\right)$ we have found families $s_{\beta \alpha}: W_{\beta \alpha} \rightarrow V_{\beta \alpha}$ and $K_{\beta \alpha} \subset W_{\beta \alpha}$ satisfying the statement of Corollary 1.2. Then, it is easy to see that the families of all $\left.t_{\beta} \circ s_{\beta \alpha}\right|_{s_{\beta \alpha}^{-1}\left(U_{\beta}\right)}$ and $K_{\beta \alpha} \cap s_{\beta \alpha}^{-1}\left(K_{\beta}\right)$ satisfy the statement.

In particular, in the proof we may proceed locally on $M$. This means that it is enough to show the corollary for a small neighbourhood $U_{y}$ of each point $y \in K$, a compact subneighbourhood $K_{y}$ of $y$ in $U_{y}$ and $L_{y}=L \cap f^{-1}\left(K_{y}\right)$.

Induction. Let $n=\operatorname{dim} M$. If $X$ is not of pure dimension, then it is near $L$ the union of finitely many pure dimensional analytic spaces $X_{i}$. The proof will be by induction on

$$
s=\max _{i} \max _{x \in L}\left\{\operatorname{dim}\left(f^{-1}(f(x)) \cap X_{i}, x\right)+n-\operatorname{dim}\left(X_{i}, x\right)\right\} .
$$

Since, by the Topological Remark, our problem is local on $M$, we may assume that $M$ is an open neighbourhood $U$ of the origin in $\mathbf{C}^{n}$.

Case 1. $X$ is pure dimensional.

Fix $x_{0} \in L \cap f^{-1}(0)$ and assume that $X$ is of pure dimension $m$ in a neighbourhood of $x_{0}$. By localizing $X$ about $x_{0}$ we may assume that $f$ is induced by a projection

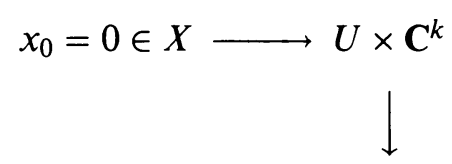

Let $r=\operatorname{dim}\left(f^{-1}(0), 0\right)$.

If $r=m-n$ there is nothing to prove. Since always $r \geq m-n$, we may assume that $s=r+n-m>0$.

Let $r<k$. Then, there exists a linear projection $p: \mathbf{C}^{k} \rightarrow \mathbf{C}^{r}$ such that

$$
\left.(f, p)\right|_{(X, 0)}:(X, 0) \rightarrow\left(U \times \mathbf{C}^{r}, 0\right)
$$

is finite. Hence the image $Z$ of $X$ by $(f, p)$ is an $m$-dimensional analytic subset of a neighbourhood of the origin in $U \times \mathbf{C}^{r}$. Since $s=r+n-m>0$, we have $(Z, 0) \neq\left(U \times \mathbf{C}^{r}, 0\right)$, and consequently there exists a nonzero function $F \in \mathscr{O}_{\left(U \times \mathbf{C}^{r}, 0\right)}$ which vanishes identically on $(Z, 0)$. In a neighbourhood of the origin we write

$$
F(y, t)=\sum_{\beta} \Delta_{\beta}(y) t^{\beta}, \quad \text { where }(y, t) \in U \times \mathbf{C}^{r} .
$$

Let $\sigma: U^{\prime} \rightarrow U$ be the blowing-up of the ideal $I$ generated by all the $\Delta_{\beta}$ (and hence by the finite number of them). Consider the following diagram

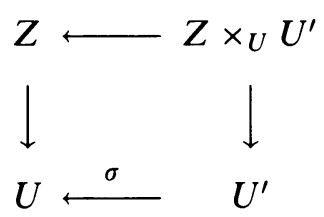

where $Z \times{ }_{U} U^{\prime} \subset U^{\prime} \times \mathbf{C}^{r}$. Let $Z^{\prime}$ denote the strict transform of $Z$ by $\sigma$. At each point $y_{0} \in U^{\prime}$ the ideal $\left(\sigma^{*} I\right)_{y_{0}^{\prime}}$ is invertible (i.e., principal and hence generated by some $\left.\Delta_{\beta_{0}} \circ \sigma\right)$. Then, near $y_{0}$

$$
F\left(\sigma\left(y^{\prime}\right)\right)=\sum_{\beta} \Delta_{\beta}\left(\sigma\left(y^{\prime}\right)\right) t^{\beta}=\Delta_{\beta_{0}}\left(\sigma\left(y^{\prime}\right)\right) \cdot F^{\prime}\left(y^{\prime}, t\right),
$$


where

$$
F^{\prime}\left(y^{\prime}, t\right)=\sum_{\beta} \Delta_{\beta}^{\prime}\left(y^{\prime}\right) t^{\beta} \quad \text { and } \quad \Delta_{\beta}^{\prime}=\frac{\Delta_{\beta} \circ \sigma}{\Delta_{\beta_{0}} \circ \sigma} .
$$

It is easy to see that $Z^{\prime} \subset F^{\prime-1}(0)$ and $F^{\prime}$ does not vanish identically on any fiber of the projection $U^{\prime} \times \mathbf{C}^{r} \rightarrow U^{\prime}$.

Let $f^{\prime}: X^{\prime} \rightarrow U^{\prime}$ be the strict transform of $f$ by $\sigma$. From the above it follows that

$$
s^{\prime}\left(x^{\prime}\right)=\operatorname{dim}\left(f^{\prime-1}\left(f^{\prime}\left(x^{\prime}\right)\right), x^{\prime}\right)+n-m<s
$$

at every point $x^{\prime} \in X^{\prime}$ corresponding to a neighbourhood of $x_{0}$.

Note that if $r=k$, then in order to obtain (1.1) we simply apply the above construction without choosing the projection $p$ (so $Z=X$ ). By the construction, (1.1) holds also for all $x^{\prime} \in X^{\prime}$ corresponding to a neighbourhood of $x_{0}$ in $L$.

Case 2. $X$ is not of pure dimension.

Then we apply Case 1 to each $X_{i} \rightarrow M$ separately, let us say by blowing up the ideal $I_{i}$. If we blow up the product $\prod I_{i}$, then each $\left(\sigma^{*} I_{i}\right)$ becomes invertible. Let $f^{\prime}: X^{\prime} \rightarrow U^{\prime}$ denote the strict transform of $f$ by this blowing up and let $X_{i}^{\prime} \subset X^{\prime}$ correspond to $X_{i}$. Then by Case 1

$$
s^{\prime}\left(x^{\prime}\right)=\max _{i} \operatorname{dim}\left(f^{\prime-1}\left(f^{\prime}\left(x^{\prime}\right)\right) \cap X_{i}, x^{\prime}\right)+n-\operatorname{dim}\left(X_{i}^{\prime}, x^{\prime}\right)<s
$$

for all the points corresponding to a neighbourhood of $x_{0}$ in $L$.

Since $L \cap f^{-1}(0)$ is compact we may cover it by a finite number of open subsets $V_{i}$, such that (1.2) holds for the points of corresponding of $V_{i}$ by blowing-up an ideal $J_{i}$. Then, by the same argument as above, the blowing-up of the ideal $\prod J_{i}$ gives (1.2) for the points corresponding to a neighbourhood of $L \cap f^{-1}(0)$ in $L$.

Smoothness of centers. The above construction yields everything we need except for the smoothness of centers. In fact in the construction above we can replace the blowing-up of a given ideal $I$ by the compositions of local blowings-up with smooth centers $\sigma$ such that $\sigma^{*} I$ is locally invertible. For instance this can be achieved by using Main Theorem II" of Hironaka [H2]. A more elementary argument can be derived from [BM1]. Namely, let $I=$ $\left(g_{i}\right)_{i=1}^{k}$. Take a family $\left\{\sigma_{\alpha}\right\}$ of compositions of local blowings-up with smooth centers satisfying the statement of Theorem 4.4 of [BM1] for all the $g_{i}$ and their differences $g_{i}-g_{j}$. Then all $g_{i} \circ \sigma_{\alpha}$ and all $g_{i} \circ \sigma_{\alpha}-g_{j} \circ \sigma_{\alpha}$ which are not identically equal to zero are locally normal crossings (see Definition 2.2 below). By the following lemma this assures that all $\sigma_{\alpha}^{*} I$ are locally invertible.

Lemma 1.3 (Bierstone and Milman [BM1, Lemma 4.7], Sussmann [S, (6.VI)]). Let $\alpha, \beta, \gamma \in \mathbf{N}^{n}$ and let $a(x), b(x), c(x)$ be invertible elements of $\mathbf{R}\{x\}$. If

$$
a(x) x^{\alpha}-b(x) x^{\beta}=c(x) x^{\gamma},
$$

then either $\alpha \leq \beta$ or $\beta \leq \alpha$ (in the lexicographic order).

This ends the proof of Corollary 1.2.

Real case. Here is a real analytic version of Corollary 1.2 (a stronger result can be obtained from [H1, 4.17] or [HLT, Théorème 4]). 
Corollary 1.4. Let $f: X \rightarrow M$ be a morphism of real analytic spaces and assume that $M$ is nonsingular. Let $L$ and $K$ be compact subsets of $X$ and $M$ respectively. Then, there exists a finite number of analytic morphisms $s_{\alpha}: W_{\alpha} \rightarrow M$ such that:

(1) each $s_{\alpha}$ is the composition of a finite sequence of real local blowings-up with smooth nowhere dense centers;

(2) for each $\alpha$ there is a compact subset $K_{\alpha}$ of $W_{\alpha}$ and

$$
\bigcup_{\alpha} s_{\alpha}\left(K_{\alpha}\right)=K
$$

$\left(3^{\prime}\right)$ the strict transforms $\tilde{f}_{\alpha}: \widetilde{X}_{\alpha} \rightarrow \widetilde{W}_{\alpha}$ of a complexification of $f$ by complexifications of $s_{\alpha}$ satisfy at every point $x \in \widetilde{X}_{\alpha}$ corresponding to $L$ the equidimensionality property:

$$
\operatorname{dim}_{\mathbf{C}}\left(\tilde{f}_{\alpha}^{-1}\left(\tilde{f}_{\alpha}(x)\right) \cap \tilde{X}_{\alpha}^{\prime}, x\right)=\operatorname{dim}_{\mathbf{C}}\left(\tilde{X}_{\alpha}, x\right)-\operatorname{dim}_{\mathbf{R}} M,
$$

for every irreducible component $\tilde{X}_{\alpha}^{\prime}$ of $\tilde{X}_{\alpha}$ at $x$.

Proof. We use a slight modification of the proof of Corollary 1.2.

Let $\tilde{f}: \widetilde{X} \rightarrow \widetilde{M}$ be a complexification of $\mathrm{f}$. If we proceed as in the proof of Corollary 1.2 , then the ideal of $\mathscr{O}_{y}$, where $y \in f(L)$, which we blow up may be choosen real (i.e., generated by complexifications of real analytic functions). Therefore, we may assume that each such blowing-up $\tilde{\sigma}: \widetilde{U}^{\prime} \rightarrow \widetilde{U}$ is a complexification of a real analytic blowing-up $\sigma: U^{\prime} \rightarrow U$. Let $\tilde{f}^{\prime}: \tilde{X}^{\prime} \rightarrow \widetilde{U}^{\prime}$ be the strict transform of $\tilde{f}$ by $\tilde{\sigma}$. Then $\tilde{X}^{\prime}$ is a complexification of real analytic space. Proceeding by induction (on the dimension of fibres of complexification of $f$ ) we consider the set $L^{\prime} \subset \widetilde{X}^{\prime}$ of real points of $\widetilde{X}^{\prime}$ corresponding to $L$. By this construction we get a family of maps $s_{\alpha}: W_{\alpha} \rightarrow M$ and their complexifications $\tilde{s}_{\alpha}: \widetilde{W}_{\alpha} \rightarrow \widetilde{M}$ satisfying (1) and (2), and $\left(3^{\prime}\right)$ at the real points $L_{\alpha}$ of $\widetilde{X}_{\alpha}$ corresponding to $L$. But then equidimensionality is satisfied at some neighbourhood of $L_{\alpha}$, so to get the result it suffices to replace $\widetilde{W}_{\alpha}$ by a small neighbourhood of $W_{\alpha}$ (in $\widetilde{W}_{\alpha}$ ).

\section{RECTILINEARIZATION OF SUBANALYTIC FUNCTIONS}

In this section we shall prove a version of the Rectilinearization Theorem for subanalytic functions (Theorem 2.7 below).

Definition 2.1. Let $U$ be an open subanalytic subset of $\mathbf{R}^{n}$. We call a function $f: U \rightarrow \mathbf{R}$ subanalytic if the closure in $\mathbf{R}^{n} \times \mathbf{P}^{1}$ of the graph $\Gamma_{f}$ of $f$ is a subanalytic subset of $\mathbf{R}^{n} \times \mathbf{P}^{1}$ (where $\mathbf{P}^{1}=\mathbf{R} \cup\{\infty\}$ denotes the real projective line).

Observe that we do not require $f$ to be continuous. One may also define subanalytic functions as those whose graphs are subanalytic only in $\mathbf{R}^{n} \times \mathbf{R}^{1}$ (see for example [DLS]). In this way one gets a broader class of functions for which our rectilinearization (Theorem 2.7 below) does not hold. Note that a function $f$ is subanalytic in our sense if and only if $f$ and its reciprocal $f^{-1}$ are subanalytic in the weaker one (see also [K1] the classes $\mathbf{S U B}(\mathbf{M})$ and $\operatorname{SUBB}(\mathbf{M}))$. Both classes coincide for $f$ locally bounded on $\mathbf{R}^{n}$. 
The sum and the product of subanalytic functions is again a subanalytic function [K1, Theorème 2.4] on the intersection of the domains and the same is true for the quotient on the set where the denominator is not zero. Each subanalytic function $f: U \rightarrow \mathbf{R}$ is analytic on an open dense subanalytic subset of $\bar{U}$.

Let us, throughout this section, denote the standard projections $\mathbf{R}^{n} \times \mathbf{P}^{1} \rightarrow$ $\mathbf{R}^{n}, \mathbf{R}^{n} \times \mathbf{P}^{1} \rightarrow \mathbf{P}^{1}$ by $\pi_{1}$ and $\pi_{2}$ respectively.

Definition 2.2. Let $M$ be an analytic manifold (over $\mathbf{K}=\mathbf{R}$ or $\mathbf{C}$ ) and let $f$ be an analytic function on $M$. We say that $f$ is locally normal crossings if each point of $M$ admits a coordinate neighbourhood $U$, with coordinates $x=\left(x_{1}, x_{2}, \ldots, x_{n}\right)$, such that

$$
f(x)=x_{1}^{\alpha_{1}} x_{2}^{\alpha_{2}} \cdots x_{n}^{\alpha_{n}} g(x),
$$

where $x \in U, \alpha_{i} \in \mathbf{N}$, and $g$ is analytic and nowhere vanishes in $U$.

We say that $f$ is normal crossings if (2.1) can be achieved in one coordinate system $\left(M=U \subset \mathbf{K}^{n}\right)$.

Note that the function which is identically equal to zero is not normal crossings. By this convention, if the product $\prod f_{i}$ of analytic functions is (locally) normal crossings, then each of $f_{i}$ is (locally) normal crossings.

By [BM1] or [H1] the continuous subanalytic functions satisfy the following property.

Proposition 2.3. Let $U$ be an open subset of $\mathbf{R}^{n}$ and let $f: U \rightarrow \mathbf{R}$ be a continuous subanalytic function. Then there exist a real analytic n-dimensional manifold $N$ and a proper surjective real analytic mapping $\phi: N \rightarrow U$ such that $f \circ \phi$ is locally normal crossings (on these components of $N$ where it does not vanish identically).

We sketch briefly the proof of Proposition 2.3. By the resolution of singularities there exist a real analytic n-dimensional manifold $M$ and a proper real analytic map $\psi: M \rightarrow \mathbf{R}^{n} \times \mathbf{P}^{1}$ whose image is just $\overline{\Gamma_{f}}$ ([BM1, Theorem 0.1$]$ or [H1, Proposition 8.1]). The function $f \circ \pi_{1} \circ \psi$ is analytic on $\psi^{-1}(U)$ and now we may apply to it the rectilinearization theorem for analytic functions ([H2, Main Theorem II"] or [BM1, Theorem 4.4]). This gives the result.

Definition 2.4. A subset $X$ of $\mathbf{R}^{n}$ is a (closed) quadrant if there is a partition of $\{1,2, \ldots, n\}$ into disjoint subsets $I_{0}, I_{-}$, and $I_{+}$, such that

$$
X=\left\{x \in \mathbf{R}^{n} ; x_{i}=0 \text { if } i \in I_{0}, x_{i} \leq 0 \text { if } i \in I_{-} \text {and } x_{i} \geq 0 \text { if } i \in I_{+}\right\} .
$$

Definition 2.5. We call a family of real analytic morphisms $s_{\alpha}: V_{\alpha} \rightarrow \mathbf{R}^{n}, \alpha \in I$, locally finite if for each compact $K \subset \mathbf{R}^{n}, s_{\alpha}^{-1}(K)=\varnothing$ for all but finite number of $\alpha$.

The rectilinearization of subanalytic sets may be obtain by maps of special form, namely the compositions of local blowings-up (see [H1, Theorem 7.1]). For closed subanalytic subsets of $\mathbf{R}^{n}$ it can be expressed as follows.

Theorem 2.6. Let $X$ be a closed subanalytic subset of $\mathbf{R}^{n}$. Then there exist a locally finite collection of real analytic morphisms $s_{\alpha}: V_{\alpha} \rightarrow \mathbf{R}^{n}$ such that each 
of them is the composition of a finite sequence of local blowings-up with smooth centers and

(1) each $V_{\alpha}$ is isomorphic to $\mathbf{R}^{n}$ and there are compact subsets $K_{\alpha}$ of $V_{\alpha}$ such that $\bigcup_{\alpha} s_{\alpha}\left(K_{\alpha}\right)$ is a neighbourhood of $X$ in $\mathbf{R}^{n}$;

(2) for each $\alpha, s_{\alpha}^{-1}(X)$ is a union of quadrants in $\mathbf{R}^{n}$.

We will show Theorem 2.6 in the course of the proof of Theorem 2.7 (see Remark 2.10 below). It is interesting to ask whether the rectilinearization of subanalytic functions may be also obtained by local blowings-up. The following simple example shows that generally this is not possible. The function $f(x)=$ $|x|^{1 / r}, r \in \mathbf{N}$, is a well defined subanalytic (even semialgebraic) function on $\mathbf{R}$ but any blowing-up can not change its domain. Note that the substitution $x= \pm y^{2 r}$ makes $f$ analytic and normal crossings.

In [BM2] Bierstone and Milman have developed a method which shows that the compositions of local blowings-up and substitution of powers give rectilinearization of any continuous subanalytic function. We shall prove below that in fact in this compositions it is enough to substitute powers only at the last step after all local blowings-up. This result we shall use in the next section to give a new proof of Theorem 1.4 of [BM2] and in [P] to construct a Lipschitz stratification of subanalytic sets.

Theorem 2.7 (Rectilinearization of subanalytic functions). Let $U$ be an open subset of $\mathbf{R}^{n}$ and let $f: U \rightarrow \mathbf{R}$ be a continuous subanalytic function. Then there exist a locally finite collection $\Psi$ of real analytic morphisms $\phi_{\alpha}: W_{\alpha} \rightarrow \mathbf{R}^{n}$ such that:

(1) each $W_{\alpha}$ is isomorphic to $\mathbf{R}^{n}$ and there are compact subsets $K_{\alpha}$ of $W_{\alpha}$ such that $\bigcup_{\alpha} \phi_{\alpha}\left(K_{\alpha}\right)$ is a neighbourhood of $\bar{U}$;

(2) for each $\alpha$ there exist $r_{i} \in \mathbf{N}, i=1, \ldots, n$, such that $\phi_{\alpha}=\sigma_{\alpha} \circ \psi_{\alpha}$, where $\sigma_{\alpha}$ is the composition of finite sequence of local blowings-up with smooth centers and

$$
\psi_{\alpha}(x)=\left(\varepsilon_{1} x_{1}^{r_{1}}, \varepsilon_{2} x_{2}^{r_{2}}, \ldots, \varepsilon_{n} x_{n}^{r_{n}}\right), \text { for some } \varepsilon_{i}=-1 \text { or } 1 ;
$$

(3) for each $\alpha: \phi_{\alpha}\left(W_{\alpha}\right) \subset \bar{U}$ and $f \circ \phi_{\alpha}$ extends from $\phi_{\alpha}^{-1}(U)$ on $W_{\alpha}$ to one of the following functions: (a) the function identically equal to zero; (b) a normal crossings; (c) the inverse of a normal crossings (this can happen only if $\left.\phi_{\alpha}(0) \in \bar{U} \backslash U\right)$;

(4) Let $\phi_{\alpha}=\sigma_{\alpha} \circ \psi_{\alpha} \in \Psi$ and $\phi_{\alpha}(0) \in U$. Then $\phi_{\alpha}\left(W_{\alpha}\right) \subset U$ and for every choice of $\varepsilon_{i}$ there is $\phi_{\beta}=\sigma_{\beta} \circ \psi_{\beta} \in \Psi$ such that $\sigma_{\beta}=\sigma_{\alpha}$ and $\psi_{\beta}$ is given by (2.2).

Proof. Let $f: U \rightarrow \mathbf{R}$ be as above and let us assume for simplicity that $U$ is relatively compact. By the resolution of singularities $([\mathrm{H} 2]$ or $[\mathrm{BM} 1$, Theorem $0.1])$ there exist a real analytic manifold $N$ and a proper real analytic map $\psi: N \rightarrow \mathbf{R}^{n} \times \mathbf{P}^{1}$ whose image is $\overline{\Gamma_{f}}$.

Apply Corollary 1.4 to $\pi_{1} \circ \phi: N \rightarrow \mathbf{R}^{n}, L=N$ and $K$ a compact neighbourhood of $\bar{U}$, and let $s_{\alpha}: W_{\alpha} \rightarrow \mathbf{R}^{n}, K_{\alpha} \subset W_{\alpha}$ be the maps and compact sets satisfying the statement of this corollary. Take one of them $s=s_{\alpha}: W=W_{\alpha} \rightarrow \mathbf{R}^{n}$. Let $\left(\widetilde{\left.\pi_{1} \circ \phi\right)^{\prime}}: \widetilde{N}^{\prime} \rightarrow \widetilde{W}\right.$ be the strict transform of a complexification of $\pi_{1} \circ \phi$. By Corollary 1.4 we may assume $\left(\widetilde{\pi_{1} \circ \phi}\right)^{\prime}$ to be a finite map. 
Consider $N^{\prime}=\overline{N \times \mathbf{R}^{n}}(W \backslash E) \subset \tilde{N}^{\prime}$, where $E$ denotes the union of the exceptional divisors. Let $\left(\pi_{1} \circ \phi\right)^{\prime}: N^{\prime} \rightarrow W, s^{\prime}: N^{\prime} \rightarrow N$ be the maps induced by the standard projections. Then, the image $\Gamma^{\prime}$ of $N^{\prime}$ in $W \times \mathbf{P}^{1}$ by the map $\left(\left(\pi_{1} \circ \phi\right)^{\prime}, \pi_{2} \circ \phi \circ s^{\prime}\right)$ is contained in a proper analytic subset of $W \times \mathbf{P}^{1}$ (since the complexification of $\left(\pi_{1} \circ \phi\right)^{\prime}$ is finite) whose projection on $W$ is finite (in the algebraic sense). Moreover, in our case, $\Gamma^{\prime}$ is just the closure of the graph of

$$
g=f \circ s: s^{-1}(U) \rightarrow \mathbf{R},
$$

so the projection $\Gamma^{\prime} \rightarrow W$ over $s^{-1}(U)$ is one-to-one.

Take any $x_{0} \in K_{\alpha}$. Choosing coordinates near $x_{0}$ we may assume that $x_{0}$ is the origin in $\mathbf{R}^{n}$. By the above $g$ satisfies near the origin an equation of the type

$$
\left(g^{k}+\sum_{i=1}^{k} a_{i} \cdot g^{k-i}\right) \cdot\left(g^{-l}+\sum_{j=1}^{l} \tilde{a}_{j} \cdot g^{j-l}\right) \equiv 0,
$$

where all $a_{i}, \tilde{a}_{j}$ are analytic in a neighbourhood of the origin and $\tilde{a}_{j}(0)=0$ for all $i$. By the Topological Remark $(\S 1)$, it is enough to show the statement for $g$ restricted to a small neighbourhood of $x_{0}$.

We consider two cases: the first when $x_{0} \in s^{-1}(U)$, so $g$ is well defined and continuous in a neighbourhood of $x_{0}$, and the second $x_{0} \in s^{-1}(\bar{U} \backslash U)$.

Case 1. $x_{0} \in K_{\alpha} \cap s^{-1}(U)$. Then (2.3) has a form

$$
g^{k}+\sum_{i=1}^{k} a_{i} \cdot g^{k-i} \equiv 0
$$

and we may assume that the discriminant $\Delta(x)$ of

$$
F(t, x)=t^{k}+\sum_{i=1}^{k} a_{i}(x) \cdot t^{k-i}, \quad t \in \mathbf{R},
$$

is not identically equal to zero.

Using Theorem 4.4 of [BM1] we may assume that both $\Delta$ and the first $a_{i}$ which is not identically equal to zero (i.e. either $a_{0}$ or $a_{1}$ ) are normal crossings. Note that the discriminant of a complexification of $F$ is a complexification of $\Delta$ and consequently is also normal crossings (in a neighbourhood of the origin).

For $\delta=\left(\delta_{1}, \ldots, \delta_{n}\right) \in \mathbf{R}_{+}^{n}$ we denote $U_{\delta}=\left\{x \in \mathbf{C}^{n}:\left|x_{i}\right|<\delta_{i}\right\}$. The following observation is due to Sussmann ( $\S 5$ of $[\mathrm{S}]$ ).

Lemma 2.8. Let $G(t, x)=t^{k}+\sum_{i=1}^{k} a_{i} \cdot t^{k-i}$ be a complex analytic function defined in $\mathbf{C} \times U_{\delta}$ and assume that the discriminant $\Delta(x)$ of $G$ is normal crossings. Then, there exist positive integers $r_{i}$ such that for any $\varepsilon_{i}=1$ or -1

$$
G\left(t, \varepsilon_{1} y_{1}^{r_{1}}, \varepsilon_{2} y_{2}^{r_{2}}, \ldots, \varepsilon_{n} y_{n}^{r_{n}}\right)=\prod_{i=1}^{k}\left(t-b_{i}(y)\right)
$$

in $\mathbf{C} \times U_{\delta^{\prime}}$, where $\delta^{\prime}=\left(\delta_{1}^{1 / r_{1}}, \ldots, \delta_{n}^{1 / r_{n}}\right)$ and $b_{i}$ are complex analytic functions (depending maybe on $\varepsilon_{i}$ ). Moreover, all the differences of $b_{i}$ are normal crossings and if the first $a_{i}$ which is not identically equal to zero is normal crossings, then the same is true for all the $b_{i}$ that are all not identically equal to zero. 
Remark. We need to consider various $\varepsilon_{i}$ since we are interested in the real domain and the images of all real maps $y \rightarrow\left(\varepsilon_{1} y_{1}^{r_{1}}, \varepsilon_{2} y_{2}^{r_{2}}, \ldots, \varepsilon_{n} y_{n}^{r_{n}}\right)$ (with fixed $r_{i}$ but all possible $\varepsilon_{i}=1$ or -1 ) cover a neighbourhood of the origin in $\mathbf{R}^{n}$.

Proof. By assumption, the projection of the zero set $Z$ of $G$ on $U_{\delta}$ is finite. Fix $i=1, \ldots, n$ and take a point $x^{0} \in H_{i}=\left\{x_{i}=0, x_{j} \neq 0\right.$ for $\left.j \neq i\right\}$. Then, by the Puiseux Lemma (with parameter) and the assumption on the discriminant we may find $r_{i}$ such that the substitution

$$
x_{i}=\varepsilon_{i} y_{i}^{r_{i}}, \quad x_{j}=y_{j} \quad \text { if } j \neq i,
$$

for all possible $\varepsilon_{i}$, gives (2.5) near $x^{0}$. Such $r_{i}$ does not depend on the choice of $x_{0} \in H_{i}$ (in the sense that if it is good at one point it is so at the others). Take such $r_{i}$ and fix also all $\varepsilon_{i}$. Then, $b_{i}$ satisfying (2.5) are well defined as a (not ordered) set of analytic functions in the complement of a subset of (complex) codimension 2 . Since this complement has to be simply connected, the $b_{i}$ are in fact well defined bounded complex analytic functions outside this set. By the Hartogs Theorem we may extend the $b_{i}$ to the whole neighbourhood $U_{\delta^{\prime}}$ of the origin which proves the first part of the lemma.

Since the discriminant of $G\left(t, \varepsilon_{1} y_{1}^{r_{1}}, \varepsilon_{2} y_{2}^{r_{2}}, \ldots, \varepsilon_{n} y_{n}^{r_{n}}\right)$ is normal crossings so are the differences of $b_{i}$. The product of all $b_{i}$ equals

$$
(-1)^{k} a_{0}\left(\varepsilon_{1} y_{1}^{r_{1}}, \varepsilon_{2} y_{2}^{r_{2}}, \ldots, \varepsilon_{n} y_{n}^{r_{n}}\right),
$$

and consequently, if $a_{0}$ is normal crossings so are all of $b_{i}$. If $a_{0} \equiv 0$, then so is exactly one of $b_{i}$, and the product of the rest of $b_{i}$ equals

$$
(-1)^{k-1} a_{1}\left(\varepsilon_{1} y_{1}^{r_{1}}, \varepsilon_{2} y_{2}^{r_{2}}, \ldots, \varepsilon_{n} y_{n}^{r_{n}}\right),
$$

which is by assumption normal crossings. This ends the proof of the lemma.

Apply the lemma to a complexification of $F$. Then there exist positive integers $r_{i}$ such that in a neighbourhood of the origin

$$
F\left(t, \varepsilon_{1} y_{1}^{r_{1}}, \varepsilon_{2} y_{2}^{r_{2}}, \ldots, \varepsilon_{n} y_{n}^{r_{n}}\right)=\prod_{i=1}^{k_{1}}\left(t-b_{i}(y)\right) \prod_{j=1}^{\left(k-k_{1}\right) / 2}\left(t-c_{j}(y)\right)\left(t-\bar{c}_{j}(y)\right),
$$

where the $b_{i}$ are real analytic functions and the $c_{j}$ are complex-valued real analytic functions. Fix $c=c_{j}=d+i \cdot e$, where $d=\operatorname{Re}(c), e=\operatorname{Im}(c)$. Then

$$
(t-c)(t-\bar{c})=(t-d)^{2}+e^{2}
$$

and since $\Delta$ is normal crossings so is $e=(c-\bar{c}) / 2 i$. Thus we have established the real analytic version of Lemma 2.8 .

Lemma 2.9. Let $F(t, x)=t^{k}+\sum_{i=1}^{k} a_{i} \cdot t^{k-i}$ be a real analytic function $(t \in \mathbf{R}$, $x$ from a neighbourhood of the origin in $\mathbf{R}^{n}$ ) and assume that the discriminant $\Delta(x)$ of $F$ is normal crossings. Then, there exist positive integers $r_{i}$ such that for any $\varepsilon_{i}=1$ or -1 and in some neighbourhood of the origin

$$
F\left(t, \varepsilon_{1} y_{1}^{r_{1}}, \varepsilon_{2} y_{2}^{r_{2}}, \ldots, \varepsilon_{n} y_{n}^{r_{n}}\right)=\prod_{i=1}^{k_{1}}\left(t-b_{i}(y)\right) \cdot \prod_{j=1}^{\left(k-k_{1}\right) / 2}\left(\left(t-d_{j}(y)\right)^{2}+e_{j}^{2}(y)\right),
$$

where $b_{i}, d_{i}$, and $e_{i}$ are real analytic functions (depending on $\varepsilon_{i}$ ). 
Moreover, all the differences of $b_{i}$ and all $e_{i}$ are normal crossings and if the first $a_{i}$ which is not identically equal to zero is normal crossings, the same is true for all $b_{i}$ which are all not identically equal to zero.

We continue the proof of Theorem 2.7. Take $r_{i}$ satisfying the statement of Lemma 2.9 for $F$ given by (2.4) and fix all $\varepsilon_{i}$. The function

$$
h(y)=g\left(\varepsilon_{1} y_{1}^{r_{1}}, \varepsilon_{2} y_{2}^{r_{2}}, \ldots, \varepsilon_{n} y_{n}^{r_{n}}\right)
$$

is continuous, real-valued, subanalytic, and satisfies the equation

$$
\prod_{i=1}^{k_{1}}\left(h-b_{i}\right) \cdot \prod_{j=1}^{\left(k-k_{1}\right) / 2}\left(\left(h-d_{j}\right)^{2}+e_{j}^{2}\right) \equiv 0 .
$$

Since all $e_{j}$ are normal crossings $\left(\left(h-d_{j}\right)^{2}+e_{j}^{2}\right)$ can vanish only on the union of coordinate hyperplanes $\mathscr{H}=\left\{y_{1} \cdot y_{2} \cdots y_{n}=0\right\}$. Therefore, since $h$ is continuous,

$$
\prod_{i=1}^{k_{1}}\left(h-b_{i}\right) \equiv 0 .
$$

This does not necessarily mean that $h$ equals one of $b_{i}$, but this is certainly true on $\mathscr{H}_{+}=\left\{y_{i} \geq 0 i=1,2, \ldots, n\right\}$ since on $\mathscr{H}_{+} \backslash \mathscr{H}$ all $b_{i}$ are distinct. Therefore, if we substitute $y_{i}=z_{i}^{2}, i=1,2, \ldots, n$ (the family of maps $z \rightarrow\left(\varepsilon_{1} z_{1}^{2 r_{1}}, \varepsilon_{2} z_{2}^{2 r_{2}}, \ldots, \varepsilon_{n} z_{n}^{2 r_{n}}\right)$ with various $\varepsilon_{i}$ cover the neighbourhood of the origin in $\mathbf{R}^{n}$ ) we get

$$
h\left(z_{1}^{2}, z_{2}^{2}, \ldots, z_{n}^{2}\right)=b_{i}\left(z_{1}^{2}, z_{2}^{2}, \ldots, z_{n}^{2}\right)
$$

for each $z$ from a neighbourhood of the origin in $\mathbf{R}^{n}$ and some $b_{i}$. This ends the proof in Case 1.

Remark 2.10. Theorem 2.6 follows from Case 1 applied to $f(x)=\operatorname{dist}(x, X)$. Case 2. $x_{0} \in K_{\alpha} \cap s^{-1}(\bar{U} \backslash U)$.

By Theorem 2.6 we may assume that $s^{-1}(\bar{U})$ is a union of quadrants. As in Case 1 we make the first not identically equal to zero $a_{i}$ and $\tilde{a}_{j}$ and the discriminants of $F(t, x)=t^{k}+\sum_{i=1}^{k} a_{i}(x) \cdot t^{k-i}$ and $\tilde{F}(t, x)=t^{-l}+\sum_{j=1}^{l} \tilde{a}_{j}(x)$. $t^{j-l}$ normal crossings. We may also do it simultaneously for $x_{1} \cdot x_{2} \cdots x_{n}$ so $s^{-1}(\bar{U})$ is still be a union of quadrants. By Lemma 2.9 applied to $F$ and $\tilde{F}$, the function $h$ we get satisfies an equation of the type

$$
\prod_{i=1}^{k_{1}}\left(h-b_{i}\right) \cdot \prod_{j=1}^{l_{1}}\left(h^{-1}-b_{j}\right) \equiv 0 .
$$

The rest of the proof is the same as in Case 1. This ends the proof of Theorem 2.7.

\section{ARC-ANALYTIC FUNCTIONS}

Let $M$ be a real analytic manifold and let $\operatorname{dim} M=n$. We call a function $f: M \rightarrow \mathbf{R}$ arc-analytic if $f \circ \gamma$ is analytic for every analytic arc $\gamma: I \rightarrow M$ (where $I=(-1,1) \subset \mathbf{R})$. This notion was introduced by Kurdyka in [K2] in 
relation with arc-symetric semialgebraic sets, where he considered only functions with semialgebraic graphs, but it in fact appeared before in the works of Kuo on blow-analytic equivalence [Ku]. Kuo considered the maps $f: X \rightarrow Y$ of real analytic siaces, which he called blow-analytic, and which become analytic after composition with modifications $X^{\prime} \rightarrow X$. He also gave a nont:ivial example $f: \mathbf{R}^{2} \rightarrow \mathbf{R}$, defined by $f(x, y)=x^{2} y /\left(x^{2}+y^{2}\right), f(0,0)=0$. It is obvious that blow-analytic functions are arc-analytic and it is interesting to ask whether the coverse is true (maybe with additional assumptions). This question was first posed by Kurdyka. Namely he asked whether an arc-analytic function with semialgebraic graph is blow-analytic. The affirmative answer was given in [BM2, Theorem 1.1] by Bierstone and Milman, where they also gave a characterization (Theorem 4.4 loc. cit.) of arc-analytic functions with subanalytic graph as blow-analytic in a weaker sense that is such functions which become analytic after compositions with local blowings-up. Here we present a proof of this result based on our rectilinearization of subanalytic functions (Theorem 2.7).

Theorem 3.1. A function $f: M \rightarrow \mathbf{R}$ is arc-analytic and has a subanalytic graph if and only if there is a locally finite family of analytic morphisms $\left\{\sigma_{\alpha}: M_{\alpha} \rightarrow M\right\}$ and compacts $K_{\alpha} \subset M_{\alpha}$ such that:

(1) $\bigcup \sigma_{\alpha}\left(K_{\alpha}\right)=M$;

(2) each $\sigma_{\alpha}$ is a composition of finitely many local blowings-up with smooth centers;

(3) each $f \circ \sigma_{\alpha}$ is analytic.

Proof. The "if" is obvious. Since the problem is local we may assume that $M=$ $U \subset \mathbf{R}^{n}$ is a small neighbourhood of the origin. By simple argument of [BM2, Lemma 6.8] each arc-analytic function with subanalytic graph is continuous. Let $\Psi=\left\{\phi_{\alpha}=\sigma_{\alpha} \circ \psi_{\alpha}\right\}$ be a family of functions satisfying the statement of Theorem 2.7 for $(f, U)$. Fix $\sigma=\sigma_{\alpha}$ and the corresponding $r_{i} \in \mathbf{N}$. Then $h=f \circ \sigma$ is arc-analytic and for each $\psi$ as given by (2.2) $h \circ \psi$ is normal crossings (in particular analytic). We shall show that it follows that $h$ is analytic.

Fix $\psi$ (and so the $\varepsilon_{i}$ ) for a moment. Let

$$
h \circ \psi(y)=\sum a_{\beta} y^{\beta} .
$$

Then on the quadrant $\mathscr{H}_{\left\{\varepsilon_{i}\right\}}=\left\{x \in \mathbf{R}^{n} ; \varepsilon_{i} \cdot x_{i} \geq 0\right.$ for $\left.i=1,2, \ldots, n\right\}$

$$
h(x)=\sum a_{\beta} \prod_{i=1}^{n}\left(\varepsilon_{i} \cdot x_{i}\right)^{\beta_{i} / r_{i}} .
$$

Suppose that for some $\beta^{0}$ one of coordinates $\beta_{i}^{0}$ is not divisible by $r_{i}$ and that $a_{\beta^{0}} \neq 0$. Then, for generic $c=\left(c_{1}, \ldots, \widehat{c_{i}}, \ldots, c_{n}\right)$, such that $\varepsilon_{j} \cdot c_{j} \geq 0$ holds for each $j \neq i$, the function

$$
\gamma(t)=h\left(c_{1}, \ldots, c_{i-1}, t, c_{i+1}, \ldots, c_{n}\right)
$$

has the Puiseux expansion with nonzero coefficient at $t^{\beta^{0} / r_{i}}$. This contradicts the assumption that $h$ is arc-analytic. Therefore, $h$ is analytic on the quadrant $\mathscr{H}_{\left\{\varepsilon_{i}\right\}}=\left\{J x \in \mathbf{R}^{n} ; \varepsilon_{i} \cdot x_{i} \geq 0\right\}$ (more precisely, it extends to an analytic function on a neighbourhood of $\left.\mathscr{H}_{\left\{\varepsilon_{i}\right\}}\right)$. 
Denote by $\left\{h_{k}\right\}$ the family of analytic functions constructed above for all quadrants $\mathscr{H}_{\left\{\varepsilon_{i}\right\}}$. We claim that they define one analytic function. If not, then there would exist two points $q_{1}, q_{2} \in \mathbf{R}^{n}$ such that in a neighbourhood of $q_{1}$ we have $h \equiv h_{k_{1}}, h\left(q_{1}\right) \neq h_{k_{2}}\left(q_{1}\right)$ and in a neighbourhood of $q_{2}$ we have $h \equiv h_{k_{2}}$ and $h\left(q_{2}\right) \neq h_{k_{1}}\left(q_{2}\right)$. But we may connect $q_{1}$ and $q_{2}$ by an analytic arc (simply a segment) and since $h$ is arc-analytic we get a contradiction. This ends the proof of the theorem.

Remark. The assumption on subanalycity of the graph is essential. An example of an arc-analytic function whose graph is not subanalytic and which is not continuous was constructed in [BMP]. A different proof of existence of arcanalytic function whose graph is not subanalytic was given by Kurdyka (private communication).

We call a function $f: M \rightarrow \mathbf{R}$ weakly arc-analytic if for each analytic arc $\gamma:[0,1] \rightarrow M$ the composition $f \circ \gamma$ is analytic in a neighbourhood of 0 . By the same argument as in [BM2, Lemma 6.8], a weakly arc-analytic function with subanalytic graph has to be continuous. Therefore by the proof of Theorem 3.1 we get

Proposition 3.2. A function $f: M \rightarrow \mathbf{R}$ is weakly arc-analytic and has a subanalytic graph if and only if there is a locally finite family of analytic morphisms $\left\{\sigma_{\alpha}: M_{\alpha} \rightarrow M\right\}$ and compacts $K_{\alpha} \subset M_{\alpha}$ such that

(1) $\bigcup \sigma_{\alpha}\left(K_{\alpha}\right)=M$;

(2) each $\sigma_{\alpha}$ is a composition of finitely many local blowings-up with smooth centers;

(3) each $M_{\alpha}$ is isomorphic to $\mathbf{R}^{n}$ and $f \circ \sigma_{\alpha}$ is continuous on $\mathbf{R}^{n}$ and analytic on each quadrant.

\section{ACKNOWLEDGMENTS}

The author would like to thank Tzee-Char Kuo, Krzysztof Kurdyka and Pierre Milman for many valuable discussions and suggestions concerning the paper, the referee for many detailed remarks and express his gratitude to the Max-Planck-Institut für Mathematik for warm hospitality and Alexander von Humboldt Stiftung for financial support during the preparation of this paper.

\section{REFERENCES}

[B] D. Barlet, Majoration du volume des fibres génériques et forme géométrique du théorème d'applatissment, C. R. Acad. Sci. Paris I Math. 288 (1979), 29-31; Lecture Notes in Math., vol. 822, Springer-Verlag, Berlin and New York, 1979, pp. 1-18.

[BM1] E. Bierstone and P. D. Milman, Semianalytic and subanalytic sets, Inst. Hautes Etudes Sci. Publ. Math. 67 (1988), pp. 5-42.

[BM2] _ Arc-analytic functions, Invent. Math. 101 (1990), 411-424.

[BMP] E. Bierstone, P. D. Milman, and A. Parusinski, A function which is arc-analytic but not continuous, Proc. Amer. Math. Soc. 113 (1991), 419-423.

[DD] J. Denef and L. van den Dries, P-adic and real subanalytic sets, Ann. of Math. (2) 128 (1988), 73-138.

[DLS] Z. Denkowska, S. Lojasiewicz, and J. Stasica, Certaines propriétés élémentaires des ensembles sous-analytiques, Bull. Polish Acad. Sci. Math. 27 (1979), 529-535. 
[H1] H. Hironaka, Introduction to real analytic sets and real-analytic maps, Istituto "L.Tonelli", Pisa, 1973.

[H2] - Resolution of singularities of an algebraic variety over a field of characteristic zero I, II, Ann. of Math. (2) 79 (1964), 109-326.

[H3] Stratification and flatness, Proc. Nordic Summer School Real and Complex Singularities (Oslo 1976), Sijthoff and Noordhoff, 1977, pp. 199-265.

[HLT] H. Hironaka, M. Lejeune-Jalabert, and B. Teissier, Platificateur local en géométrie analytique et aplatissement local, Astérisque 7-8 (1973), 441-463.

[K1] K. Kurdyka, Points reguliere d'un sous-analytique, Ann. Inst. Fourier (Grenoble) 38 (1988), 133-156.

[K2] - Ensembles semi-algébriques symétriques par arc, Math. Ann. 282 (1988), 445-462.

[Ku] T.-C. Kuo, On classification of real singularities, Invent. Math. 82 (1985), 257-262.

[P] A. Parusiński, Lipschitz stratification of subanalytic sets, Ann. École Norm. Sup. (to appear).

[S] H. J. Sussmann, Real-analytic desingularization and subanalytic sets: an elementary approach, Trans. Amer. Math. Soc. 317 (1990), 417-461.

School of Mathematics and Statistics, University of Sydney, Sydney, NSW 2006, AusTRALIA

E-mail address: parusinski_a@maths.su.oz.au 